\section{INCREASING MHC-I EXPRESSION TO POTENTIATE IMMUNE CHECKPOINT BLOCKADE THERAPY}

${ }^{1}$ Shengqing Gu*, 'Wubing Zhang, 'Xiaoqing Wang, ${ }^{1}$ Peng Jiang, ${ }^{3}$ Nicole Traugh, 'Ziyi Li, ${ }^{1}$ Clifford Meyer, ${ }^{4}$ Blair Stewig, ${ }^{1}$ Yingtian Xie, ${ }^{1}$ Xia Bu, ${ }^{1}$ Michael Manos, ${ }^{1}$ Alba Font-Tello, ${ }^{1}$ Evisa Gjini, ${ }^{1}$ Ana Lako, ${ }^{1}$ Klothilda Lim, ${ }^{1} J a k e$ Conway, ${ }^{1}$ Alok Tewari, 'Zexian Zeng, 'Avinash Das Sahu, ${ }^{1}$ Collin Tokheim, 'Jason Weirather, ${ }^{1}$ Jingxin Fu, ${ }^{1} Y i$ Zhang, ${ }^{1}$ Benjamin Kroger, ${ }^{5}$ Jin Hua Liang, ${ }^{1}$ Paloma Cejas, ${ }^{1}$ Gordon Freeman, ${ }^{6}$ Scott Rodig, ${ }^{1}$ Henry Long, ${ }^{6}$ Benjamin Gewurz, ${ }^{1} F$. Stephen Hodi, ${ }^{1}$ Myles Brown, ${ }^{1} X$ Shirley Liu. ${ }^{1}$ DanaFarber Cancer Institute, Boston, MA, United States; ${ }^{2}$ National Cancer Institute, Bethesda, MD, United States; ${ }^{3}$ Tufts University, Boston, MA, United States; ${ }^{4}$ Boston Children's Hospital, Boston, MA, United States; ${ }^{5}$ Brigham and Women's Hospital, Boston, MA, United States; ${ }^{6}$ Brigham and Women's Hospital, Boston, MA, United States

Background Cancer immunotherapy, especially immune checkpoint blockade (ICB) therapy, is leading to a paradigm shift in cancer treatment, as a small percentage of cancer patients have obtained durable remission following ICB treatment. Successful ICB responses rely on cancer cells presenting antigens to the cell surface via the major histocompatibility complex (MHC), which activates antigen-specific T-lymphocytes to kill cancer cells. Type-I MHC (MHC-I) is wildly expressed in all cell types and mediates the interaction with cytotoxic CD8 T cells. However, over $65 \%$ of cancer patients are estimated to show defects in MHC-I-mediated antigen presentation, including downregulation of its expression that can lead to primary or acquired resistance to ICB therapy, and therapeutic strategies to effectively restore or boost MHC-I are limited.

Methods Here, we employed a CRISPR screening approach with dual-marker FACS sorting to identify factors that decouple the regulation of MHC-I and PD-L1. The experimentally validated target was used to generate a KO differential expression signature. Using this signature, we analyzed transcriptome data from drug perturbation studies to identify drugs that regulate MHC-I but not PD-L1. Finally, we validated the effect of the identified drug to enhance ICB response in a T-celldependent manner in vivo.

Results CRISPR screens identified TRAF3, a suppressor of the NF- $\mathrm{BB}$ pathway, as a negative regulator of MHC-I but not PD-L1. The Traf3-knockout (Traf3-KO) gene expression signature is associated with better survival in ICB-naive cancer patients and better ICB response. We then screened for drugs with similar transcriptional effects as this signature and identified SMAC mimetics. We experimentally validated that the SMAC mimetic birinapant upregulates MHC-I, sensitizes cancer cells to T-cell-dependent killing, and adds to ICB efficacy. However, in cancer cells with high NF-KB activity, the effect of birinapant on MHC-I is weak, indicating context-dependent MHC-I regulation.

Conclusions In summary, Traf3 deletion specifically upregulates MHC-I without inducing PD-L1 in response to various cytokines and sensitizes cancer cells to T-cell-driven cytotoxicity. The SMAC mimetic birinapant phenocopies Traf3-knockout and sensitizes MHC-I-low melanoma to ICB therapy. Further studies are needed to elucidate the context-dependencies of MHC-I regulation. Our findings provide preclinical rationale for treating some tumors expressing low MHC-I with SMAC mimetics to enhance sensitivity to immunotherapy. The approach used in this study can be generalized to identify other drugs that enhance immunotherapy efficacy.

Acknowledgements This study was supported by grants from the NIH (R01CA234018 to XSL, R01AI137337 to BEG, P50CA101942-12 and P50CA206963 to GJF), Breast Cancer Research Foundation (BCRF-19-100 to XSL), Burroughs Wellcome Career Award in Medical Sciences (to BEG), and Sara
Elizabeth O'Brien Trust Fellowship (to SG).We thank Drs. Kai Wucherpfennig and Deng Pan for their insightful suggestions on this study.

Ethics Approval All mice were housed in standard cage in Dana-Farber Cancer Institute Animal Resources Facility (ARF). All animal procedures were carried out under the ARF Institutional Animal Care and Use Committee (IACUC) protocol and were in accordance with the IACUC standards for the welfare of animals.

http://dx.doi.org/10.1136/jitc-2021-SITC2021.700 